\section{Affiliated Organizations}

\section{AMERICAN ASSOCIATION OF PETROLEUM GEOLOGISTS (AAPG)}

\section{President}

Robert D. Cowdery

Suite 1007

107 N. Market Street

Wichita, KS 67202-1815

Tel: +1 3162679030 or

c/o AAPG Headquarters

P.O. Box 979

Tulsa, OK 74101

USA

\section{Executive Director \\ Lyle F. Baie \\ AAPG Headquarters \\ 1444 South Boulder Ave. \\ P.O. Box 979 \\ Tulsa Oklahoma 74101-0979 \\ USA \\ Tel: + l 9185842555 \\ Fax: + 19185602652 \\ E-mail: lbaie@aapg.org}

\section{AMERICAN GEOLOGICAL INSTITUTE (AGI)}

\section{President}

Dr. Edward C. Roy, Jr.

Trinity University

715 Stadium Drive

San Antonio. TX 78212-0001

USA

Tel: +12107368201

\section{Executive Director}

Marcus E. Milling

American Geological Institute

4220 King St.

Alexandria, VA 22302-1507

USA

Tel: +17033792480

Fax: + 17033797563

E-mail: mmilling@agiweb.org

\section{AMERICAN GEOPHYSICAL UNION (AGU)}

\section{President}

Sean C. Solomon

Carnegie Institution of Washington

Department of Terrestrial Magnetism

5241 Broad Branch Road, NW

Washington, DC 20015

USA

Tel: 202/686-4370 Ext. 4444

Fax: 202/364-8726

E-mail: scs@dtm.ciw.edu

\section{Executive Director}

A.F. Spilhaus, Jr.

2000 Florida Ave. N.W.

Washington, DC 20009

USA

Tel: +12024626900

Fax: +12023280566

E-mail: fspilhaus@kosmos.agu.org

\section{ARAB GEOLOGISTS ASSOCIATION}

President

Mr. Ibrahim T. Oraibi

Union of Libyan Geologists

P.O. Box 10208

Tripoli

LIBYA

Fax: +218214901160

Secretary General

Dr. Wissan S. Al-Hashimi

Arab Geologists Association

P.O. Box 1247

Baghdad

REPUBLIC OF IRAQ

Tel: +96415375314

Fax: +96417193532

\section{Vice President}

Mr. Sideeq A. Kadar

General Union of Sudanese Geologists

P.O. Box 410

Khartoum

SUDAN

Tel: +24911471393

Fax: +24911463341

THE ASSOCIATION OF EXPLORATION OF GEOCHEMISTS (AEG)

\section{President}

William B. Coker

BHP Minerals Canada Ltd.

33 Yonge Street, Suite 610

Toronto, Ontario K1A 0E8

CANADA

Tel: +4163683884

Fax: +4163650763

\section{Secretary}

Sherman P. Marsh

US Geological Survey

PO Box 25046, MS 973

Denver Federal Center

Denver $\mathrm{CO}$

USA 80225

Tel: +13032365521

Fax: +1 3032363200

E-mail: smarsh@helios.cr.usgs.gov

\section{Vice Presidents}

David L. Garnett (Australia)

E-mail: bq@atom.ansto.gov.au

Peter R. Simpson (United Kingdom)

E-mail: p.simpson@bgs.ac.uk

Treasurer

Gwendy E. Hall (Canada)

E-mail: hall@gsc.emr.ca

\section{ASSOCIATION OF EUROPEAN GEOLOGICAL SOCIETIES}

\section{President}

Professor V.A. Glebovitsky

Russian Academy of Sciences

Makarova emb., 2

St. Petersburg, 199034

RUSSIAN FEDERATION

Tel: +8122184701 or 2960673

Fax: +8122184801

Telex: 121.419 MEOBR SU
Executive Secretary

Dr B. Deffontaines

Univ. Pierre et Marie Curie

Dept. of Geotectonics

T26-0, 1E, Case 129

4, Place Jussieu

F75252 Paris, Cedex 05

FRANCE

Tel: +33144275830

Fax: +33 I 44275085

E-mail: bd@lgs.jussieu.fr

ASSOCIATION OF GEOSCIENTISTS FOR INTERNATIONAL DEVELOP. MENT (AGID)

President

Dr. S. D. Limaye

Director, Groundwater Institute

2050 Sadashiv Peth

Vijayanagar Colony

Pune 411030

INDIA

Tel: +91212441262

Fax: +91212477712

Telex: Dr Limaye c/o 1457525 VMJC IN

\section{Secretary}

Dr. A. J. Reedman

British Geological Survey

Keyworth

Nottingham NG12 5GG

UK

Tel: +441159363465

Fax: +44 II5936 3474

E-mail: a.reedman@bgs.ac.uk

\section{Vice Presidents}

For Africa

Tunde Adegbesan (Nigeria)

Prof T C Davies (Kenya)

For Asia

Ms. Afia Akhtar (Bangledesh)

For Latin America

Dr. Arlei Macedo (Brazil)

For Developed Countries

Dr. Naz Ahmed Shaihk (Sweden)

AGID Headquarters

Ms. Katia Maria Mellito

Institute of Geosciences

University of Sao Paulo

CP 11.348

05422-970 Sao Paulo

BRAZIL

Tel: +55118184206

Fax: +55112104958

e-mail: kmellito@usp.br

\section{Treasurer}

Ms. Fiona Fordyce

British Geological Survey

Keyworth

Nottingham NG12 5GG

UK

Tel: +44 1159363465

Fax: +44 1159363474 
ASSOCIATION INTERNATIONALE POUR I'ETUDES DES ARGILES (AIPEA)

\section{President}

Haydn H. Murray

Department of Geological Sciences

Indiana University

1005 East Tenth Street

Bloomington, IN 47405

USA

Tel: +18128555583

Fax: +18128557899

E-mail: murrayh@indiana.edu

\section{Secretary General}

Robert A. Schoonheydt

Centrum voor Oppervlaktechemie en Katal-

yse (COK)

K.U. Leuven

K. Mercierlaan 92

3001 Heverlee

BELGIUM

Tel: +3216321592

Fax: +32 16321998

E-mail: Robert.Schoonheydt@agr.kuleuven.ac.be

\section{Vice President}

Elen Roaldset (Norway)

\section{Past President}

Jose M. Serratosa (Spain)

\section{Treasurer}

Pam M. Huang (Canada)

\section{CARPATHIAN BALKAN GEOLO- GICAL ASSOCIATION}

\section{President}

Dr. Werner R. Janoschek

Geological Survey of Austria

Rasumofskygasse 23

P.O.B. 127

A - 1031 Vienna

AUSTRIA

Tel: +4317125674 , ext 43

Fax: +431712567456

E-mail: wjanoschek@cc.geolba.ac.at

\section{Secretary General}

Dr. Michael Wagreich

Institute of Geology

Geo-Center

University of Vienna

Althanstr. 14

A - 1090 Vienna

AUSTRIA

Tel: +43131336, ext 1695

Fax: +43131336782

E-mail: michael.wagreich@univie.ac.at

COMMISSION FOR THE GEOLOGICAL MAP OF THE WORLD (CGMW)

\section{President}

Prof. Jean Dercourt

Dept. Géologie Sedimentaire

Université; Paris VI

Tour 15 éme étage, 4 Place Jussieu F-75252

Paris Cedex 45

FRANCE

Tel: +33144273751

Fax: +331442738 31
Secretary General

Dr. Philippe Bouysse

Maison de la Geologie

77 rue Claude Bernard

F-75005 Paris

FRANCE

Tel: +33147072284

Fax: +33143367655

Telex: CGMW $206411 \mathrm{~F}$

\section{CIRCUM-PACIFIC COUNCIL FOR \\ ENERGY AND MINERAI \\ RESOURCES (CPCEMR)}

\section{Chairman}

Dr. Michel T. Halbouty

The Halbouty Center

5100 Westheimer Road

Houston, TX 77056

USA

Tel: +17136221130

Fax: + l 7136225360

\section{Executive Director}

Dr. John A. Reinemund

P.O. Box 890

Leesburg, VA 22075

USA

Tel: +1 7037771491

Fax: +1 7037774463

\section{Circum-Pacific Map Project}

\section{General Chairman \\ Dr. George Gryc \\ U.S. Geological Survey \\ 345 Middlefield Road, MS 952 \\ Menlo Park, CA 94025-3591 \\ USA \\ Tel: +1 4153294002 \\ Fax: +14153294013 \\ Telex: 403929}

\section{EUROPEAN ASSOCIATION OF SCI- ENCE EDITORS (EASE)}

\section{President}

Dr. David W. Sharp

The Lancet, 42 Bedford Square

London, WC1B 3SL.

UK

Fax: +44 1713236441

E-mail: dw.sharp@elsevier.co.uk

\section{Secretary-Treasurer \\ Mrs. Jennifer T. Gretton \\ P.O. Box 426 \\ Guildford, GU4 7ZH \\ UK \\ E-mail: secretary@ease.org.uk}

\section{THE GEOCHEMICAL SOCIETY (GS)}

\section{President}

Dr. Michael Drake

University of Arizona

Space Science Building \#92

Tucson

AZ 85721

USA

Tel: +15206216962

Fax: +1 5206214933

E-mail: drake@Ipl.arizona.edu

\section{Secretary}

David J. Wesolowski

Oak Ridge National Lab

Chemistry Division MS 6110

P.O. Box 2008

Oak Ridge, TN 37831-6110

USA

Tel: + 14235746903

Fax: + 4235744961

E-mail: dqw@ornl.gov

\section{International Secretary}

Ross S. Taylor

Nuclear Physics R.S. Pys. S.

Australian National University

Canberra

ACT 2601

AUSTRALIA

Tel: + 6162492089

Fax: +6162490748

E-mail: gill.taylor@anu.edu.au

\section{Treasurer}

Donald Elthon

Ocean Sciences/Marine Geol. Geophys.

National Science Foundation

4201 Wilson Blvd.

Arlington, VA 22230

USA

Tel: +17033061586

Fax: +17033060390

E-mail: delthon@nsf.gov

\section{GEOLOGICAL SOCIETY OF AFRICA}

President

Prof. S. Muhongo

Department of Geology

University of Dar-es-Salaam

P.O. Box 35052

Dar-es-Salaam

TANZANIA

Tel: +2555143638

Fax: +2555143380

E-mail: muhongo@unidar.gn.apc.org

\section{Secretary General}

Dr. S.N. Ayonghe

Department of Geology

University of Buea

P.O. Box 63

Buea

CAMEROON

Tel: +237322134

Fax: +237322272

\section{GEOLOGICAL SOCIETY OF AMERICA (GSA)}

\section{President}

Eldridge M. Moores

Department of Geology

University of California - Davis

Davis, CA 95616-8605

USA

Tel: +19167520352

\section{Executive Director}

Donald M. Davidson. Jr.

Geological Society of America

3300 Penrose Place

P.O. Box 9140

Boulder, CO 80301-9140

USA

Tel: +13034472020

Fax: +13034471133 and 4470648

E-mail: davidson (ogeosociety.org 


\section{INTERNATIONAL ASSOCIATION OF ENGINEERING GEOLOGY}

\section{President}

Prof. Paul Marinos

National Technical University of Athens

42. Patission St

10682 Athens

GREECE

\section{Secretary General}

Dr. Louis Primel

Lab. Central des Ponts et Chaussées

58. Boulevard Lefébvre

F- 75732 Paris Cedex 15

FRANCE

Tel: $+33140435243 / 44$

Fax: +33 । $40435498 / 99$

E-mail: primel@Icpc.inrets.fr

\section{INTERNATIONAL ASSOCIATION OF GEOMORPHOLOGISTS (IAG)}

\section{President}

Prof. Dr. Dietrich Barsch

Dept. of Geography

University of Heidelberg

Im Neuenheimer Feld 348

D-69120 Heidelberg

GERMANY

Tel: $+49622156+570$

Fax: +496221564996

E-mail: J71@VM.URZ.UNI-Heidelberg.DE

\section{Secretary}

Dr. Robert J. Allison

Dept. of Geography

University of Durham

Durham DHI 3LE

UK

Tel: +44913742458

Fax: +44913742456

E-mail: R.J.Allison@uk.ac.durham

Vice President

Prof. Olav Slaymaker (Canada)

\section{Treasurer}

Prof. Vic. R. Baker (USA)

\section{Publication Secretary}

Prof. Avijit Gupta (Singapore)

\section{INTERNATIONL ASSOCIATION OF GEOCHEMISTRY AND COSMO- CHEMISTRY (IAGC)}

\section{President}

Dr. Gunter Faure

Dept. of Geology and Mineralogy

Ohio State University

Columbus, Ohio 43210-1398

USA

Tel: + I 6142923454

Fax: +16142927688

E-mail: bheath@magnus.acs.ohio-state.edu

\section{Secretary}

Mel Gascoyne

AECL Research

Whiteshell Laboratories

Pinawa, Manitoba ROE ILO

CANADA

Tel: +12047532311 ext. 2207

Fax: + 12047532703

E-mail: GASCOYNEM@wl.aecl.ca
Past President

Dr. Hitoshi Sakai (Japan)

\section{Treasurer}

Dr. David T. Long (USA)

INTERNATIONAL ASSOCIATION ON
THE GENESIS OF ORE DEPOSITS (IAGOD)

\section{President}

Ian R. Plimer

School of Earth Sciences

University of Melbourne

Parkville, Victoria 3052

AUSTRALIA

Tel: +61393446520

Fax: +61393446520

E-mail: ian_plimer@muwayf.unimelb.edu.au

\section{Secretary General}

Jaroslav Aichler

Czech Geological Survey

P.O.Box 65

79001 Jesenik

CZECH REPUBLIC

Tel: +426453294

Tel: +426353092 (Home)

Fax: +426829907

E-mail: aichler@telecom.cz

\section{Treasurer}

Richard I. Grauch

U.S. Geological Survey

MS 973, Federal Center

Denver, CO 80225

USA

Tel: +1 3032365551

Fax: +13032363200

E-mail: rgrauch@helios.cr.usgs.gov

\section{Membership Secretary}

Dr. Reimar Seltmann

Geoforschungszentrum Potsdam

Telegrafenberg A50)

D-14473 Potsdam

\section{GERMANY}

Tel.: +493312881433

Fax.: +493312881436

E-mail: seltm@gfz-potsdam.de

\section{Past President}

Prof. Rongfu Pei (China)

\section{INTERNATIONAL ASSOCIATION OF HYDROGEOLOGISTS (IAH)}

\section{President}

Prof. Michael Knight

National Centre for Groundwater Management

University of Technology

PO Box 123. Broadway NSW 2007

AUSTRALIA

Tel: +61295141984

Fax: +61295141985

E-mail:

Groundwater.Management@uts.edu.au

\section{Secretary General}

Dr. Andrew Skinner

P.O. Box 9

Kenilworth CV8 IJG

UK

Tel/Fax: +44926 56561

Telex: 336748 NRAST G

E-mail:100074.1445@compuserve.com

\section{Vice Presidents}

Prof. Jacques Avias (France)

Prof. John M. Sharp (USA)

Dr. Jaroslav Vrba (Czech Republic)

\section{Treasurer}

Dr. Wilhelm Struckmeier

BGR, Stilleweg 2

GERMANY

Tel: +495116433301

Fax: +495116433684

E-mail: BZ20STRUCK@bzm401.hannover.bgr.de

\section{Past President}

Dr. John E. Moore (USA)

E-mail: moore123@aol.com

\section{INTERNATIONAL ASSOCIATION FOR MATHEMATICAL, GEOLOGY (IAMG)}

\section{President}

Dr. Ricardo A. Olea

Kansas Geological Survey

1930 Constant Avenue, Lawrence, KS 66047-2598

USA

Tel: +1 9138643965

Fax: + 19138645317

E-mail:

ricardo_olea@msmail.kgs.ukans.edu

\section{Secretary General}

Thomas A. Jones

Exxon Production Research Co.

P.O. Box 2189

Houston, TX 77252-2189

USA

Tel: +17139663046

Fax: +17139666336

E-mail: tom.a.jones@exxon.sprint.com

\section{Past President}

M.E. Hohn

West Virginia Geological and Economic Survey

P.0. Box 879

Morgantown, WV 26507-0879

USA

Tel: +1 3045942331

Fax: + 13045942575

E-mail: HOHN@GEOSRV.WVNET.EDU

\section{INTERNATIONAL ASSOCIATION OF SEDIMENTOLOGISTS (IAS)}

\section{President}

Prof. A. Bosellini

Istituto di Geologio

Universitá di Ferrara

Corso Ercole d'Este 32

44100 Ferrara

ITALY 
General Secretary

Prof. A. Strasser

Institut de Geologie

Université de Fribourg

Perolles 1700 Fribourg

SWITZERLAND

Tel: +41 26.3008978

Fax: +41263009742

E-mail: Andreas.Strasser@unifr.ch

\section{Vice Presidents}

Prof. E. F. McBride (USA)

Prof. A. M'Rabet (Tunisia)

Prof. S. Ordonez (Spain)

\section{Treasurer}

Di. I. Cojan (France)

\section{Past President}

Prof. G.G. Zuffa (Italy)

\section{INTERNATIONAL ASSOCIATION OF STRUCTURAL/TECTONIC GEOLO- GISTS (IASTG)}

\section{Coordinator}

Dr. Susan H. Treagus

Department of Geology

University of Manchester

Manchester MI3 9PL

UK

Tel: +44612753822

Fax: +44162753947

E-mail: iastg@man.ac.uk

\section{INTERNATIONAL CENTER FOR TRAINING AND EXCHANGES IN THE GEOSCIENCES}

\section{President}

Mr. Jacques Giri

SEED

130. boulevard Caméé

92240 Malakoff

FRANCE

Tel: +3301 55580500

Fax: +330155580505

E-mail: 100627.2215.compuserve.com

\section{Director}

Mr. Jean-Claude Napias

CIFEG

3 , avenue Claude-Guillemin

B.P. 6517

45065 Orléans Cedex 2

FRANCE

Tel: +330238643367

Fax: +330238643472

E-mail: jc.napias@brgm.fr

\section{Pan-African Network for a Geological} Information System (PANGIS)

\section{Coordinator}

Mrs. Marielle Arregros-Rouvreau CIFEG

3. avenue Claude-Guillemin

B.P. 6517

45065 Orléans Cedex 2

FRANCE

Tel: +330238643659

Fax: +330238643472

E-mail:m.arregros@brgm.fr
INTERNATIONAL FEDERATION OF

PALYNOLOGICAL SOCIETIES (IFPS)

\section{President}

Prof. Owen K. Davis

Department of Geosciences

University of Arizona

Tucson. Arizona 85721

USA

Tel: +15206217953

Fax: + 5206212672

E-mail: palynolo@geo.arizona.edu

\section{Secretary-Treasurer}

Dr. R. Scott Anderson

Center for Environmental Sciences and Education

Box 5694

Northern Arizona University

Flagstaff. A.Z 86011-5694

USA

Tel: +1 5205235821 (office)

Tel: + $1520774-9062$ (home)

Fax: + 15205237423

E-mail: sa@nauvax.ucc.nau.edu

\section{Newsletter Editor}

Fredrick J. Rich

Department Geology and Geography

Landrum Box 8149

Georgia Southern College

Statesboro, GA 30460-8149

USA

Tel: +19126815361 or 6810849

Fax: +19126810196

E-mail: frich@gsaix2.cc.Gasou.edu

INTERNATIONAL MINERALOGICAL ASSOCIATION (IMA)

\section{President}

Stefano Merlino

Dipartimento di Scienze della Terra

Universita di Pisa

Via Santa Maria, 53

56100 Pisa

ITALY

Tel: +3950568203

Fax: +395040976

E-mail: merlino@dst.unipi.it

\section{Secretary}

Stefan S. Hafner

Institute of Mineralogy

University of Marburg

35032 Marburg

GERMANY

Tel: +496421285617

Fax: +496421288919

Telex: 482372 unr d

E-mail: hafner@mailer.uni-marburg.de

\section{Vice Presidents}

A.J. Naldrett (Canada)

E-mail: ajn@quartz.geology.utoronto.ca

Johan Zdenek (France)

E-mail: z.johan@ brgm.fr

\section{Treasurer (Interim)}

Cornelis Klein

E-mail: cklein@unm.edu
Past President

Xie Xiande (China)

INTERNATIONAL PALAEONTOLOGICAL ASSOCIATION (IPA)

President

Prof. J.A. Talent

Centre of Ecostratigraphy and Paleobiology

School of Earth Sciences

Macquarie University 2109

AUSTRALIA

E-mail: jtalent@laurel.oes.mq.edu

\section{Secretary General}

Prof. D.L. Bruton

Paleontologisk Museum

Universitet i Oslo

Sarsgate 1, Tøyen

N-0562 Oslo

NORWAY

Tel: +4722851668

Fax: +4722851810

\section{INTERNATIONAL PERMAFROST ASSOCIATION}

\section{President}

Prof Cheng Guodong

Director

Lanzhou Institute of Glaciology and

Geocryology

Chinese Academy of Sciences

Lanzhou. 730000

CHINA

Fax: +869318885241

E-mail: gdcheng@ns.lzb.ac.cn

Secretary General

Dr. Jerry Brown

P.O. Box 7

Woods Hole

MA 02543

USA

Tel: +15085481.348

Fax: + 5084571548 (Temporary. 1996)

E-mail: jerrybrown@ipc.apc.org

Vice Presidents

Dr. H. M. French

E-mail: hfrench@science.ottawa.ca

Prof. N. N. Romanovskii

E-mail: nromanovsky@glas.apc.org

\section{INTERNATIONAL SOCIETY FOR ROCK MECHANICS (ISRM)}

\section{President}

Prof. Shunsuke Sakurai

Dept. of Architecture and Civil Engineering Kobe University

Rokko-dai 1-1, Nada-ku, Kobe 657

JAPAN

Tel: +81788826260

Fax: +81788031050

E- mail: ssakurai@icluna.kobe-u.ac.jp

\section{Secretary General}

Dr. José Delgado Rodrigues

Laboratório Nacional de Engenharia Civil

101 Av. do Brasil

P-1799 Lisboa Codex

PORTUGAL

Tel: +3511848213!

Fax: +35118478187

E- mail: delgado $(\hat{)} \operatorname{lnec} . p t$ 
Regional Vice Presidents

Africa: Dr. Nielen van der Merwe (South Africa)

Asia: Dr. Ou Chin-Der (Taipei, China) Australasia: Mr. Garry R. Mostyn (Australia)

Europe: Prof. Giovanni Barla (Italy)

North America: Prof. Herbert H. Einstein (USA)

South America: Prof. Michel L. Van Sint Jan F. (Chile)

Vice Presidents at Large

Prof. Sun Jun (China)

Prof. John A. Hudson (United Kingdom)

INTERNATIONAL SOCIETY OF SOIL MECHANICS AND FOUNDATION ENGINEERING (ISSMFE)

\section{President}

Prof. M.B. Jamiolkowski

Via Ripamonti 89

20141 Milano

ITALY

Tel: +3925696160

Fax: +3925691845

\section{Secretary General}

Dr. R.H.G. Parry

University Engineering Department

Trumpington Street

Cambridge CB2 1PZ

UK

Tel: +441223355020

Fax: +44 1223359675

\section{Past President}

Prof. N.R. Morgenstern (CANADA)

Vice Presidents

Prof. M.K. El-Ghamrawy (Egypt)

Prof. A.S. Balasubramaniam (Thailand)

Mr. M.C. Ervin (Australia)

Prof. Dr. Ir. W.F. Van Impe (Belgium)

Dr. Victor Milligan (Canada)

Mr. Luis A.P. Valenzuela (Chile)

Representing:

Africa

Asia

Australasia

Europe

North America

South America

\section{INTERNATIONAL UNION FOR QUA- TERNARY RESEARCH (INQUA)}

\section{President}

Prof. Stephen C. Porter

Quaternary Research Center

AK-60

University of Washington

Seattle. WA 98195

USA

Tel: + I 2065431166

E-mail: scporter@u.washington.edu

\section{Secretary}

Prof. Sylvi Haldorsen

Agricultural University of Norway

Department of Soil and Water Sciences

P.O. Box 5028

$\mathrm{N}-1432$ Aas

NORWAY

Tel: +4764948252

Fax: +4764948211/4664948211

E-mail: sylvi.haldorsen@ijvf.nlh.no

\section{Secretariat}

Ms. Gerry Kroon

Netherlands Instit. of Appl. Geoscience-TNO

Secretariat Dept. Geo-Mapping NITG-TNO

P.O. Box 157

2000 AD Haarlem

THE NETHERLANDS

Tel: $+31(0) 235300261$

Fax: +31 (0) 235367064

E-mail: g.kroon@nitg.tno.nl

\section{Treasurer}

Dr. Ed. de Mulder (The Netherlands)

\section{Past President}

Prof. Liu Tungsheng (China)

Vice Presidents

Prof. Yoko Ota (Japan)

Prof. Timothy C. Partridge (South Africa)

Prof. Nicholas J. Shackleton (Great Britain

\section{THE METEORITICAL SOCIETY (MS)}

\section{President}

Dr. Dieter Stoeffler

Museum fuer Naturkunde

der Humboldt-Univiversitaet Berlin

Invalidenstrasse 43

D-10115

GERMANY

\section{Secretary}

Dr. Monica M Grady

The Natural History Museum

Cromwell Road

London SW7 5BD

UK

Tel: +44719389445

Fax: +44 719389268

E-mail: M.Grady@NHM.AC.UK

\section{SOCIETY OF ECONOMIC GEOLO- GISTS (SEG)}

\section{President}

Richard L. Nielsen

Queenstake Resources

13741 Braun Drive

Golden, CO 80401

USA

Tel: +13032793118

\section{Executive Director}

John A. Thoms

Little Creek One Building

5808 South Rapp Street, Suite 209

Littleton, CO 80120-1942

USA

Tel: +13037970332

Fax: +1 3037970417

E-mail: socecongeol@csn.net
SOCIEY FOR GEOLOGY APPLIED TO MINERAL DEPOSITS (SGA)

\section{President}

Professor Eugen Stumpf

Institute of Geological Sciences

Mining University

Franz Josef Strasse 18

8700 Leoben

AUSTRIA

Tel: +433842402451

Fax: +43384247016

E-mail : stumpfl@grz08,unileoben.ec.at

\section{Secretary General}

Dr. Maurice Pagel

CREGU - BP 23

F - 54501 Vandoeuvre-lés-Nancy

FRANCE

Tel: +330383441900

Fax: +330383440029

Telex: $960934 \mathrm{~F}$

E-mail: pagel@cregu.cnrs-nancy.fr

\section{Vice President}

Dr. Jan Pasava (Czech Republic)

\section{Treasurer}

Prof. Peter Herzig (Germany)

E-mail: herzig@mineral.tu-freiberg.de

\section{Regional Vice Presidents}

N. America: David Leach (USA)

$S$. America: Waldo Vivallo (Chile)

Asia: Hidehiko Shimazaki (Japan)

Australia: Philip Seccombe (Australia)

S. Africa: Hartwig Frimmel (South Africa)

\section{SOCIETY FOR SEDIMENTARY GEOLOGY (SEPM)}

\section{President}

Dr. Richard Moiola

Society for Sedimentary Geology

1731 E. 71 st Street

Tulsa, OK 74136-5108

USA

\section{Executive Director}

Cathleen P. Williams

SEPM

1731 E. 71 st Street

Tulsa, OK 74136

USA

Tel: 8008659765 (North America Only)

Tel: +19184933361 , ext. 24

Fax: + 19184932093

E-mail: cpwmsx@galaxy.galstar.com 


\section{Associated Members}

\section{AUSTRALIAN COUNCIL OF CHAIR- MAN OF EARTH SCIENCES}

Attn: Prof. Ian R. Plimer, President

Department of Geology

University of Newcastle

Rankin Drive

Newcastle, NSW 2308

AUSTRALIA

\section{AUSTRALIAN GEOLOGICAL SUR- VEY ORGANIZATION}

Bureau of Mineral Resources, Geology and Geophysics

P.O. Box 378

Canberra, ACT 2601

AUSTRALIA

\section{NORTHERN TERRITORY GEOLOGI- CAL SURVEY}

Attn: Dr. Paul Le Messurier, Director Department of Mines and Energy Centrepoint Towers Building, The Mall G.P.O. Box 2901

Darwin. NT 0801

AUSTRALIA
UNIVERSIDADE FEDERAL DO PARA

Attn: Mr. M. El-Robrini

Cx Postal 1611-Av. Perimental S/n

66000 Belem, PA

BRAZIL

\section{GEOSCIENCE RESEARCH DIVISION KDMIPE}

Attn: Dr. Jagadish Pandey, General Manager Oil \& Natural Gas Commission

9 Kaulagarh Road

Dehra Dun - 248195

INDIA

\section{DIRECTORATE OF INTERNATIONAL COOPERATION}

Attn: Dr. Fahad Huraib, Director

King Abdulaziz City for Science and Tech-

nology

P.O. Box 6086

Riyadh 11442

SAUDI ARABIA

\section{THE GEOLOGICAL SOCIETY OF SOUTH AFRICA}

Attn: Mrs. E. Burger, Secretary

P.O. Box 44283

2104 Linden

SOUTH AFRICA

\section{SLOVAK BUREAU OF GEOLOGY}

Attn: Dr. Osvald Trilek

P.O. Box 62-Bukarestska 4

81005 Bratislava 15

THE SLOVAK REBUBLIC

\section{Dr. G.A. Mori}

Viale Ugo Ojotti 23

50137 Firenze

ITALY

\section{GEOCARTO INTERNATIONAL CENTER}

Attn: Dr. K.N. Au, Research Director G.P.O. Box 4122

HONG KONG

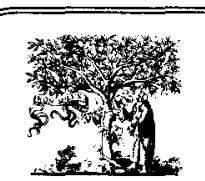

ELSEVIER

SCIENCE

\section{Miocene Stratigraphy}

An Integrated Approach

Edited by A Montanari, Osservatorio Geologico di Coldigioco. Frontale di Apiro, Italy; G.S. Odin. UniverditéPierre et Marie Curie. Lab. De Géochronologie et Sédimentologie, Paris, France and R. Coccioni, Universitá degli Studi, Instituto di Geologia, Urbino, Italy

Developments in Palaeontology and Stratigraphy Volume 15

Contents:

Part A. The Historical Stratotypes.

Part B. Geology of the Two Main Study Areas.

Part C. Studies Relevant to the Lower Miocene Subseries.

Part D. Studies Relevant to the Middle Miocene Subseries

Part E. Integrated Studies Relevant to the Upper Miocene Subseries

Part F. Miocene geochronology: methods, techniques, and results. Chronostratigraphy of Miocene stages: a proposal for the definition of precise boundaries. References. Subject index.

\section{(1997694 pages}

ISBN 0-444-82498-7 Hardbound

Publication: May 1997

Price: NLG 395.00/US\$247.00

\section{ELSEVIER SCIENCE}

European and Japanese customers are to pay in Dutch Guilders (NLG). All oth(ers pay in US Dollars (US\$)
}

\section{Geodynamic Domains in the Alpine-Himalayan Tethys}

Chief editor: Anshu K. Sinha

Editors: F.P. Sassi, D. Papnikolaou

\section{Objective of the project:}

- Study the geodynamics of Tethyan domains in Palaeozoic and their subsequent Mesozoic and Cenozoic evolution

- Elaborate on the topic of Pre-Alpine-Himalayan basement complex, basinal configuration, accretion of terrane following collision

- Understand and conceptualize the genesis and distribution of mineral deposits on the entire Tethyan zone from the Altas Mountains of Algeria to the Himalaya and Tibet situated in the extreme eastern Asian end

1997/ 81-204-1091-2/ 458 pages/ Rs. 950.00

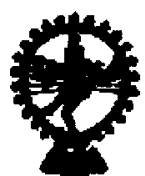

Oxford \& IBH Publishing co. Pvt. Ltd. 66 Janpath, New Delhi 110001 , India Phone: (011) 3324578,3320518 Fax: (011) 3713275,3322639 E-mail: Oxford.publ@axcess.net.in 